\title{
Analysis of Human-Machine Cooperative Robot and haptic interaction for stroke rehabilitation
}

\author{
Zhiqin Qian ${ }^{1, a}$, Tingting Jiang ${ }^{1, b}$, Yifan Liu $^{1, c}$ and Zhuming $\mathrm{Bi}^{2, \mathrm{~d}}$ \\ ${ }^{1}$ School of Mechanical and Energy Engineer, East China University of Science and Technology, \\ Shanghai 200237, China \\ ${ }^{2}$ Department of Engineering, Purdue University of Fort Wayne, Fort Wayne, Indiana 47907, USA \\ acynthiajiang520@163.com, b ${ }^{\mathrm{b}}$ qianzhiqin@ecust.edu.cn, ${ }^{\mathrm{C}}$ Yfliu54@163.com, ${ }^{\mathrm{d}}$ biz@ipfw.edu
}

\begin{abstract}
Many kinds of actuator-based haptic robots have been developed and utilized in rehabilitation to improve and strengthen stroke patient's motor capability. Now a new concept of HMCR (humanMachine Cooperative Robot) is proposed in this article, which works with the joint efforts of human user and the machine or neither respectively. However, these systems seem to be not qualitative currently. Therefore, this paper presents a quantitative analysis on the statics of the haptic robot system.
\end{abstract}

Key words: Human-machine cooperative robot, haptic interface, stroke treatment

\section{Introduction}

Stoke has been the most important in leading disability and the second to death [1]. Patient always has symptoms with paralysis, asynergia, or hemiplegia. Stroke greatly affects people daily life, which not only lowers the living quality of the patients but also increase the financial burden to the patients and their families. Proper rehabilitation treatment and training can help them regain partially or totally the motor control ability.

Researches mainly focus on physic rehabilitation and robotic rehabilitation for stroke treatment. However the robot in rehabilitation is different from the traditionally industrial robot. Traditional one only works when the structure is driven by acutators. By contrast, the robot used in rehabilitation has the function been achieved through actuators driving, users moving, or the joint effort, not just focus on one method. Distinguished from the traditional robot, a HMCR with two or three driving ways is developed in rehabilitation area. Based on the HMCR Phantom Omni, this article quantitatively analyze the interactive system between human user and robot, the Human-machine interactive platform are shown

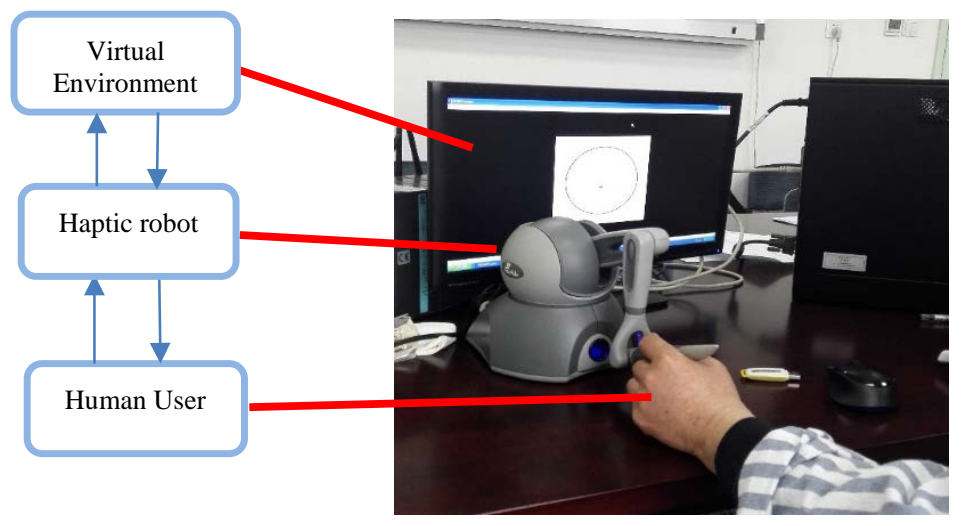

as Fig.1.

Fig. 1 A virtual environment haptic robot rehabilitation system 
There have been many studies on the system of Fig. 1. Only a few studies are focused on the quantitative analysis of haptic systems. , including (1) the haptic robotic system (Fig. 1), (2) the mapping between the virtual world and haptic robot (Fig. 1), (3) the mapping between the haptic robot and human (Fig. 1). [2] has studied Problem (1) but the work is incorrect in that the work considers the haptic robotic system alone without the presence of both the human user and the virtual world. As such, the work leads to the conclusion that the haptic robot (Fig. 1) has five degrees of freedom and performs the analysis of the five degrees of freedom robot only. This paper reports our work that was aimed at provides a quantitative analysis of the haptic robotic system under the presence of the human user as well as the presence of the virtual environment. A paper before particularly focus on the kinematics of haptic robots in the entire system. This paper especially presents the static force analysis.

\section{Haptic Interaction Platform}

A haptic interface is an actuated, computer controlled and instrumented device that allows a human user to touch and manipulate objects either within a virtual environment (VE) or in a real world through a slave of a teleoperated system such as for surgical robotics [3]. The haptic interface ensures bilateral interactions between the user and the VE in a haptic rendering process as shown in Figure 1 [4]. This dual way property, in other words being not only an input interface but also a feedback source for the user, gives a unique characteristic to the haptic device.

Virtual environment, haptic device, human user interactive system can be basically divided into two parts, the Virtual World and the Physical World.

\subsection{Physical World}

The physical world refers to the haptic device and the user in this platform. In this part the structure and kinematics of the haptic Phantom Omni device are presented (Fig. 2). The six DOFs machine have three active joints O A B respectively controlled by three motors and three passive joints C D P. The Phantom is only actuated in the first three joints.

A forward kinematic model of position (Equation 1) that establish the relations between the position of the end-effector and the joint coordinates can then be expressed as

$$
{ }_{6}^{0} T={ }_{1}^{0} T{ }_{2}^{1} T_{3}^{2} T_{4}^{3} T_{5}^{4} T{ }_{6}^{5} T=\left[\begin{array}{cccc}
n x & \text { ox } & \text { ax } & p x \\
n y & \text { oy } & \text { ay } & p y \\
n z & o z & a z & p z \\
0 & 0 & 0 & 1
\end{array}\right]
$$

$\mathrm{p}_{x}, \mathrm{p}_{y}, \mathrm{p}_{z}$ are the coordinates of the point $\mathrm{P}$, It is noted that the Phantom Omni has a built in software system for forward kinematics and kinetics, which means that the interacting force between the tip and the finger is calculated by the software system[5]. The first $3 \times 3$ matrix is the orientation of the endeffector.

\subsection{Virtual World}

Kadlecek studied several APIs (Application Program Interfaces) that is available for the design of a haptic virtual environment [6]. CHAI3D, OpenHaptics, H3D API, and others were compared. The compare of three popular haptic development frameworks are listed below. Dmitiry states that H3D API is of great simplicity and availability, which not only allows quickly prototyping in VE but allows a developer to define the whole virtual environment scene with a camera set, lights, primitive objects, complex meshes, textures within XML nodes [7]. Moreover, it is an open source which does good 
contributions to researchers. From the point of analysis and verification, exact data logging support is welcomed in haptic technology for the HMCRs.

Table. 1 Comparison of the Most Popular Haptic Development Framework

\begin{tabular}{|l|c|c|c|}
\hline & Chai 3D & H3D API & OpenHaptics \\
\hline Open Source & Yes & Yes & No \\
\hline Cross Platform & Yes & Yes & Yes \\
\hline API manual & Yes & Yes & Yes \\
\hline Examples & Yes & Yes & Yes \\
\hline Data logging support & No & Yes & Yes \\
\hline
\end{tabular}

\section{Static Force Analysis}

My letter before described a kinematic relationship for haptic robotic system including forward and inverse kinematics. However, only a research on the above aspect will not be sufficient in creating a control system for a mechanism, and a static force analysis is necessary in this work. This paper contribute to do a detailed analysis on statics.

In this paper, the haptic robot has three active joints who are under actuation and three passive joints. The three passive ones lead to a lack of sufficient known conditions for positive static analysis. From a new point of view, two methods are proposed in statics analysis of human-computer interaction on three driving torque: (1) balance principle of static force and static moment, (2) virtual work principle.

\subsection{Balance Principle of Static Force and Static Moment}

The gravity of links are not considered. We annotate the contact force and moment are $\vec{f}\left(f_{x}, f_{y}, f_{z}\right)$, $\vec{n}\left(n_{x}, n_{y}, n_{z}\right)$ between the end effector and environment. Establishing the linkage coordinate system based on D-H parameters method, the static force and static moment applied for a single linkage are shown in Fig.3.

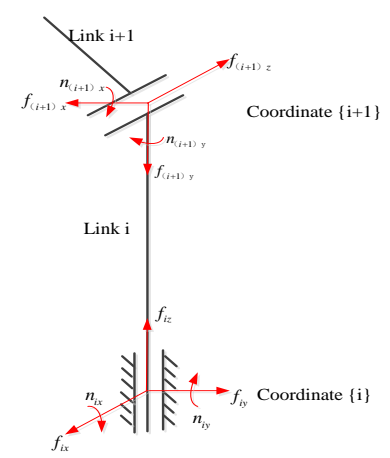

Fig. 2 Force analysis of single link

For this linkage, we got the six equilibrium equations of force and torque as $\sum \vec{f}_{i}=0$ and $\sum \vec{n}_{i}=0$. For torque $\vec{n}_{i}$, we have two equations in space because torque along the rotation axis of i not equals to 0 . After transformation, we derive equilibrium equations below:

${ }^{i} f_{i}={ }_{i+1}^{i} R^{i+1} f_{i+1}$ ${ }^{i} n_{i}={ }_{i+1}^{i+1} R^{i+1} n_{i+1}+{ }^{i} P_{i+1} \times{ }^{i} f_{i}$

For each linkage, we have the analysis as above. Next we can easily derive the unknown ones.

\subsection{Virtual Work Principle}


Assuming that the system is in balance, and there exists a minimal displacement (called Virtual displacement). We have the knowledge that the work of Cartesian space equals to the work of joint space. That is

$$
\mathrm{F} \cdot \delta \mathrm{x}=\tau \cdot \delta \theta
$$

Learning from the Introduction to Robotics and Jacobian matrix, I derived that

$$
\tau=J^{T} F
$$

Where: JT is the Jacobian in force domain, which equals to the transpose of Jacobian in postion field. $\tau$ is a $6 \times 1$ joint vector in joint space, $\mathrm{F}$ is a $6 \times 1$ force-torque vector in Cartesian space. Here we got an equation:

$$
\left(\begin{array}{l}
\tau_{1} \\
\tau_{2} \\
\tau_{3} \\
\tau_{4} \\
\tau_{5} \\
\tau_{6}
\end{array}\right)=\boldsymbol{J}^{T}\left(\begin{array}{l}
F_{R x} \\
F_{R y} \\
F_{R z} \\
M_{R x} \\
M_{R y} \\
M_{R z}
\end{array}\right)
$$

We have the assumption that $\mathrm{MRx}=\mathrm{MRy}=\mathrm{MRz}=0$ and $\tau_{1}, \tau_{2}, \tau_{3}$ are known which should be found from the V-Robot. $\tau_{4}, \tau_{5}, \tau_{6}, F_{R x}, F_{R y}, F_{R z}$ six unknown variables can be easily obtained from the six equations above.

\section{Experiment and Result}

Implement the analysis of kinematics and statics of haptic robot system, a program is written in H3D interactive software based on these two relationships, then an experiment is arranged to verify this the effectiveness of haptic interactive system.

In this experiment, patients with upper limb muscle injury and dysfunction are guided to carry out appropriate rehabilitation training through the tactile robot Phantom Omni. When the patient is training in the virtual environment, the robot will give him appropriate assistance, which can improve the enthusiasm and initiative of the patient in the rehabilitation process, and ultimately enhance the rehabilitation effect of patients. Based on the above haptic interaction system, a hypotheses is necessary that the active control rehabilitation system is effective to help stroke patients with upper limb rehabilitation.

To verify hypotheses above, 12 patients are selected. The training time of each patient is 4 mins and the training process is divided into two following stages with both 2mins: (1) Patients are required to follow

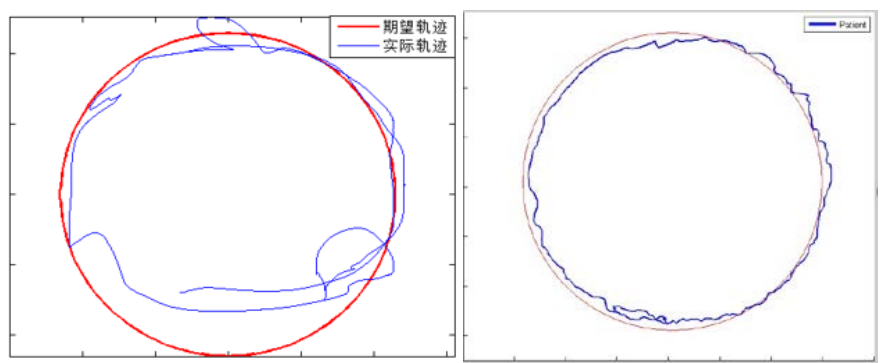

$\begin{array}{ll}\text { (a) Without assistive control } & \text { (b) With assistive control }\end{array}$

Fig. 3 Tracking trajectory with/without assistive control

the circle on screen with the stick (no force feedback). (2) Patients are required to follow the circle on screen with the stick while the device gives force feedback. When the cursor on screen are out of the predetermined trajectory, the device will drive the user's upper limb to follow the prescribed trajectory. 
The result of the effect in fulfill the tasks are shows in Fig. 4.To evaluate the work of the patients, four parameters are listed to indicate the rehabilitation performance of the stroke patient during the training process, such as the number of tracking circles $\mathrm{N}$, the average velocity $\mathrm{MV}$, error or motion accuracy error, average force MF applied to the virtual surface.

Through the paired sample tests, the first three parameters obviously show a significant difference between

the situations with and without assistive control. The numbers of circles drawn and average velocity increase with errors decrease. Thus it's evident to verified the effectiveness of assistive control haptic robot system. However, the average force don't have an significant difference.

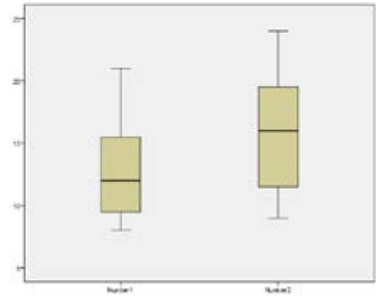

(a) Number

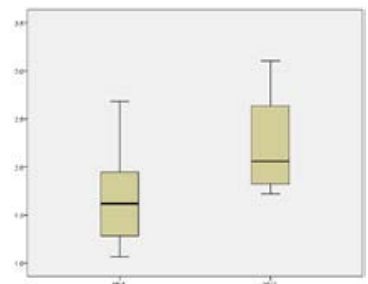

(b) MV

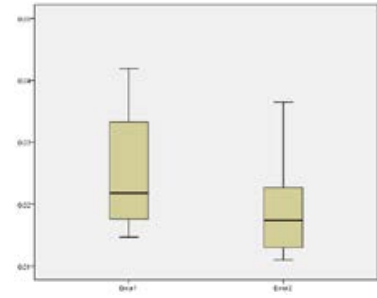

(c) Error

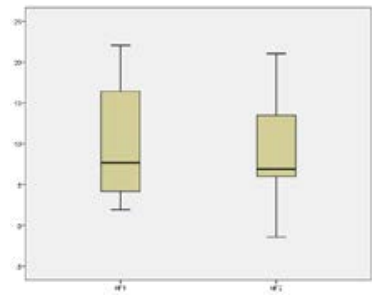

(d) $\mathrm{MF}$

Fig. 4 Evaluated parameters with and without assistive control

\section{Conclusion}

This paper comes up with a new idea of HMCR for the haptic interface robot. A static force analysis based on kinematics is proposed for HMCR Phantom Omni device ,which is applied to stroke patient therapy, With analysis of kinematics and statics, an experiment is arranged and the result shows the effectiveness of assistive control haptic robot system which do a good contribution of HMCR in rehabilitation.

\section{Acknowledgments}

This work was financially supported by Science and Engineering CRD Project of Canada, HumanMachine Production Systems (No.12410709900) and National Natural Science Foundation of China (No. 51375166.)

\section{Reference}

[1] Antonija Mitrovic, Moffat Mathews, Stellan Ohlsson, Jay Holland, Audrey McKinlay. ComputerBased Post-Stroke Rehabilitation of Prospective Memory [J]. Journal of Applied Research in Memory and Cognition, 2016, 5: 204-214.

[2] Longjiang Zhou, Kai Keng Ang, Chuanchu Wang, Kok Soon Phua, Cuntai Guan. A novel hand strength assessment method integrated into haptic knob for stroke rehabilitation [J]. IEEE Computational Intelligence in Rehabilitation and Assistive Technologies, 2013: 1-4.

[3] A. Bardorfer, M. Munih, A. Zupan, and A. Primozic, "Upper Limb Motion Analysis Using Haptic Interface", IEEE/ASME Transactions on Mechatronics, Vol. 6 No. 3, pp. 253-260, 2001

[4] E. Samur, "Systematic evaluation methodology and performance metrics for haptic interfaces", in IEEE World Haptics Conference, 2011, pp. 1-1.

[5] D. Wang, J. Li and C. Li, "An Adaptive Haptic Interaction Architecture for Knee Rehabilitation Robot", Proceedings of the IEEE International Conference on Mechatronics and Automation, 2009, pp. 
84-89.

[6] J. Laut, F. Cappa, O. Nov and M.Porfiri, "Increasing Patient Engagement in Rehabilitation Exercises Using Computer-Based Citizen Science ", Plos One, Vol. 10, No. 3, 2015.

[7] K. Bark, E. Hyman, F. Tan, E. Cha, S. A. Jax, L. J. Buxbaum and K. J. Kuchenbecker, " Effects of Vibrotactile Feedback on Human Learning of Arm Motions", IEEE Transactions on Neural Systems and Rehabilitation Engineering, Vol. 23 No. 1, pp. 51-63, 2015. 